\title{
Avaliação do uso de injetáveis em portadores de Diabetes Mellitus tipo 1 acompanhados em ambulatório de referência
}

\section{Evaluation of the use of injectables in patients with Type 1 Diabetes Mellitus accompanied in outpatient reference}

Priscila Macêdo Fernandes ${ }^{1}$. Luana Pontes Vasconcelos Lima ${ }^{1}$. Annelise Barreto de Carvalho ${ }^{2}$. Luciana Felipe Ferrer Aragão ${ }^{3}$. Milena Silva Sousa ${ }^{4}$. Renan Magalhães Montenegro Junior ${ }^{5}$. Ana Paula Dias Rangel Montenegro ${ }^{6}$.

1 Residente de Endocrinologia Pediátrica, Hospital Universitário Walter Cantídio (HUWC), Fortaleza, Ceará, Brasil. 2 Doutorado em Saúde da Criança e do Adolescente, Médica assistente e Preceptora, Ambulatório de Endocrinologia Pediátrica, Hospital Universitário Walter Cantídio (HUWC), Fortaleza, Ceará, Brasil. 3 Mestrado em Pediatria, Médica assistente e Preceptora, Ambulatório de Endocrinologia Pediátrica, Hospital Universitário Walter Cantídio (HUWC), Fortaleza, Ceará, Brasil. 4 Mestranda em Ciências Médicas, Médica preceptora, Ambulatório de Endocrinologia Pediátrica, Hospital Universitário Walter Cantídio (HUWC), Fortaleza, Ceará, Brasil. 5 Doutorado em Clínica Médica, Professor Associado da Faculdade de Medicina, Universidade Federal do Ceará (UFC), Gerente de Ensino e Pesquisa dos Hospitais Universitários (UFC/EBSERH), Fortaleza, Ceará, Brasil. 6 Doutorado em Ciências Médicas, Médica assistente e Preceptora, Ambulatório de Endocrinologia Pediátrica, Hospital Universitário Walter Cantídio (HUWC), Fortaleza, Ceará, Brasil.

\section{RESUMO}

Objetivo: Avaliar as práticas de injetáveis em pacientes com diabetes mellitus tipo 1 (DM1) acompanhados em hospital terciário. Metodologia: pacientes portadores de DM1 e seus responsáveis responderam questionário que envolvia perguntas referentes à doença e ao seu tratamento. Foram utilizados o coeficiente de correlação de Spearman, teste qui-quadrado de Pearson e teste de Kruskal-Wallis. Resultados: 119 pacientes; 0,8\% lactentes, $12,7 \%$ pré-escolares, $17,8 \%$ escolares, $64,4 \%$ adolescentes e $4,2 \%$ adultos; predomínio do sexo masculino (56,3\%). A idade ao diagnóstico do DM1 foi de 7,9 anos e tempo de doença de 3,6 anos. Média de $\mathrm{HbA1c}$ de 8,84\% com correlação positiva ao tempo de doença $(\mathrm{r}=0,226, \mathrm{p}=0,021)$. Dose total de insulina foi de $0,96 \mathrm{UI} /$ $\mathrm{kg} / \mathrm{dia}$, sendo $0,55 \mathrm{UI} / \mathrm{kg} / \mathrm{dia}(57,2 \%)$ em forma basal. Análogos de insulina de ação lenta foram utilizados em $65,5 \%$ dos indivíduos e de ação ultrarrápida em $68,1 \%$. Foi verificada maior dose de insulina total e bolus nos adolescentes ( $\mathrm{p}=0,024, \mathrm{p}=0,007)$. A média de reutilização da agulha para aplicação foi de 7,6 vezes. Enquanto a lipo-hipertrofia foi diagnosticada pelo médico em 47,8\% dos pacientes, apenas 34,7\% destes referiam sua presença. Conclusão: Esse estudo permitiu conhecimento sobre uso de insulina em pacientes com DM1 e seus resultados irão estimular melhores práticas relacionadas à saúde desses pacientes.

Palavras-chave: Diabetes Mellitus tipo 1. Insulinas. Educação em saúde.

\section{ABSTRACT}

Objective: To evaluate the injectable practices of patients with Type 1 Diabetes Mellitus (DM1) followed at a tertiary hospital. Methodology: patients with DM1 and their caregivers answered a questionnaire that involved questions related to the disease and its treatment. The Spearman correlation coefficient, Pearson's chi-square test and Kruskal-Wallis test were used. Results: 119 patients; $0.8 \%$ of infants, $12.7 \%$ of preschool children, $17.8 \%$ of school children, $64.4 \%$ of adolescents and $4.2 \%$ of adults; predominance of males (56.3\%). The age at diagnosis of DM1 was 7.9 years and disease time was 3.6 years. Mean HbA1c of $8.84 \%$ with positive correlation to disease time $(\mathrm{r}=0.226, \mathrm{p}=0.021)$. Total insulin dose was $0.96 \mathrm{IU} / \mathrm{kg} / \mathrm{day}$, being $0.55 \mathrm{IU} / \mathrm{kg} /$ day (57.2\%) in basal form. Slow-acting insulin analogues were used in $65.5 \%$ of the individuals and of ultrafast action in $68.1 \%$. A higher total insulin and bolus dose was observed in adolescents $(p=0.024, p=0.007)$. The average reuse of the needle for application was 7.6 times. While lipohypertrophy was diagnosed by the physician in $47.8 \%$ of the patients, only $34.7 \%$ of them reported their presence. Conclusion: This study allowed knowing about the use of insulin in patients with DM1 and its results will stimulate better practices related to the health of these patients.

Keywords: Diabetes Mellitus, type 1. Insulins. Health education.

Autor correspondente: Priscila Macêdo Fernandes, Rua Capitão Francisco Pedro, 1290, Rodolfo Teófilo, Fortaleza, Ceará. CEP: 60430-380. Telefone: +55 85 3366-8619. E-mail: priscilamfernandes@outlook.com

Conflito de interesses: Não há qualquer conflito de interesses por parte de qualquer um dos autores.

Recebido em: 03 Dez 2017; Revisado em: 07 Fev 2018; Aceito em: 01 Mar 2018. 


\section{INTRODUÇÃO}

O diabetes mellitus tipo 1 (DM1) é uma patologia crônica, potencialmente grave, que necessita de cuidados intensivos com orientações médicas adequadas e suporte multiprofissional desde o início. ${ }^{1,2} \mathrm{O}$ paciente diabético deve ser estimulado ao autocuidado da sua doença a partir do seu diagnóstico, com orientações quanto ao manejo da insulina, locais e formas de aplicação, noções de assepsia, descarte de insulina e seus insumos, além de conhecimento básico sobre mecanismos de ação dos diversos tipos de insulina., ${ }^{1,3}$

A educação em diabetes faz parte do cuidado integral do paciente, envolvendo interativamente o indivíduo acometido, sua família e o educador. ${ }^{2}$ Estudos demonstram que pacientes acompanhados sistematicamente por equipe multidisciplinar têm melhor prognóstico da doença com menor número de complicações agudas e crônicas. ${ }^{3}$

Esse processo educativo foi elaborado mais efetivamente na década de 90 pelo Diabetes Control and Complication Trial - DCCT, mostrando que pacientes com esquema intensivo de tratamento insulínico apresentavam menor número de complicações microvasculares, através de boas práticas de aplicação da insulina e de acompanhamento com equipe educadora multiprofissional. , $^{2,4}$

Em 2016, foi publicado o maior estudo sobre técnica de injetáveis em pacientes diabéticos realizado mundialmente após aplicação do questionário das técnicas de injeção (ITQInjection Technique Questionnaire). ${ }^{6,7}$ Em 2017, a Sociedade Brasileira de Diabetes, com base nesses resultados, publicou um posicionamento sobre tratamento dos injetáveis em pacientes diabéticos para melhor assistência ao portador de DM1. ${ }^{8}$

Diante disso, esse estudo tem como objetivo avaliar as características dos pacientes com DM1 acompanhados em serviço de referência de endocrinologia pediátrica, bem como aspectos de sua técnica de injeção.

\section{MATERIAL E MÉTODOS}

Trata-se de um estudo transversal realizado no período de maio de 2017 a agosto de 2017, no qual pacientes portadores de DM1 acompanhados em ambulatório de endocrinologia pediátrica e seus responsáveis responderam questionário que envolvia perguntas referentes à doença (idade ao diagnóstico, tempo de DM1) e ao tratamento insulínico (dose de insulina, tipo de insulinas, meio de aplicação, tamanho da agulha de aplicação, frequência de uso de agulha de aplicação, motivo para reutilização de agulhas, rodízio e grupos musculares de aplicação, assepsia, realização de prega subcutânea para aplicação, presença de lipo-hipertrofia, local de descarte de material). Os indivíduos selecionados também eram questionados sobre o horário de aplicação da insulina rápida ou ultrarrápida em relação à alimentação e sobre autoaplicação insulínica. Todo o questionário foi elaborado tendo como base o ITQ (Injection Technique Questionnaire).
O diagnóstico de diabetes foi realizado através dos critérios da American Diabetes Association. ${ }^{1,9} \mathrm{O}$ paciente era classificado como portador de DM1 através de sintomas típicos e necessidade de insulina sem interrupção desde o início do quadro. ${ }^{10}$

Os pacientes selecionados foram divididos em grupos etários de acordo com classificação da Organização Mundial de Saúde (OMS): lactente (menores que 2 anos de idade), pré-escolar (2 aos 5 anos e 11 meses), escolar (6 aos 9 anos e 11 meses), adolescente (10 a 18 anos e 11 meses), adultos (acima de 19 anos de idade cronológica). ${ }^{4}$

Durante visita médica, avaliou-se estatura (Standard Deviation Score (SDS)), peso ( $\mathrm{kg}$ ) e índice de massa corporal de cada paciente (SDS), como também a presença ou não de lipohipertrofia. Essa lesão se caracteriza por regiões nodulares formadas por gordura e tecido fibroso, hipoestésicas, na qual é observada absorção inadequada de insulina.

Esses dados foram relacionados ao resultado laboratorial da última hemoglobina glicosilada (HbA1C) de cada paciente, realizada pelo método cromatografia líquida de alta perfomance.

Foram excluídos do estudo pacientes com diagnóstico de DM1 que estavam em uso de sistema de infusão contínuo de insulina, pacientes diabéticos não tipo 1 (monogênico, tipo 2, gestacional) e aqueles que se recusaram a responder o questionário ou que não compreendiam o que era perguntado.

Os pacientes não foram colocados em risco pelo estudo. Consentimento livre e esclarecido foi obtido de todos pacientes. Esse estudo seguiu normas de pesquisa clínica e preservou preceitos da declaração de Helsinky. O estudo foi previamente aprovado pelo Comitê de Ética com parecer número: 2.437.088.

Os dados foram apresentados em média e desvio-padrão para variáveis escalares. Para as variáveis categóricas, os resultados foram expostos em frequência e taxa de prevalência de modo a investigar associações entre os dados do questionário.

Para análise das características dos grupos foi utilizado o teste U de Mann-Whitney além do teste de Kruskal-Wallis, condicionado à aderência dos dados à distribuição gaussiana. Adotou-se um nível de significância de 5\%. Na investigação de associação entre as variáveis, utilizou-se teste de qui-quadrado de Pearson e teste exato de Fisher para variáveis categóricas. As análises estatísticas foram realizadas utilizando o programa estatístico Statistical Package for the Social Sciences (SPSS), versão 22.0 (USA) e software R 3.3.1.

\section{RESULTADOS}

Foram avaliados 119 pacientes, sendo $1(0,8 \%)$ lactente, 15 $(12,7 \%)$ pré-escolares, $22(17,8 \%)$ escolares, $76(64,4 \%)$ adolescentes e $5(4,2 \%)$ adultos. Houve predominância do sexo masculino $(56,3 \%)$ em relação ao feminino.

A idade ao diagnóstico do DM1 foi de 7,9 anos (DP: $\pm 4,1$ ) e o tempo de doença de 3,6 anos (DP: $\pm 3,3$ ) (Tabela 1). Foi 
observada uma média de hemoglobina glicosilada (HbA1c) de $8,84 \%(\mathrm{DP}= \pm 2,16)$ com correlação positiva ao tempo de doença $(r=0,226 ; p=0,021)$ (Figura 1$)$. Não houve relação entre HbA1c e SDS IMC ( $p=0,461)$. A média de HbA1c foi maior nos adultos e menor nos pré-escolares $(10,19 \% \pm 2,3$ vs $7,85 \pm 0,99, p=0,186)$. Não houve, portanto, diferença estatística na comparação entre os resultados de HbA1c e os grupos etários (Tabela 1).

Figura 1. Relação entre HbA1c e tempo de doença.

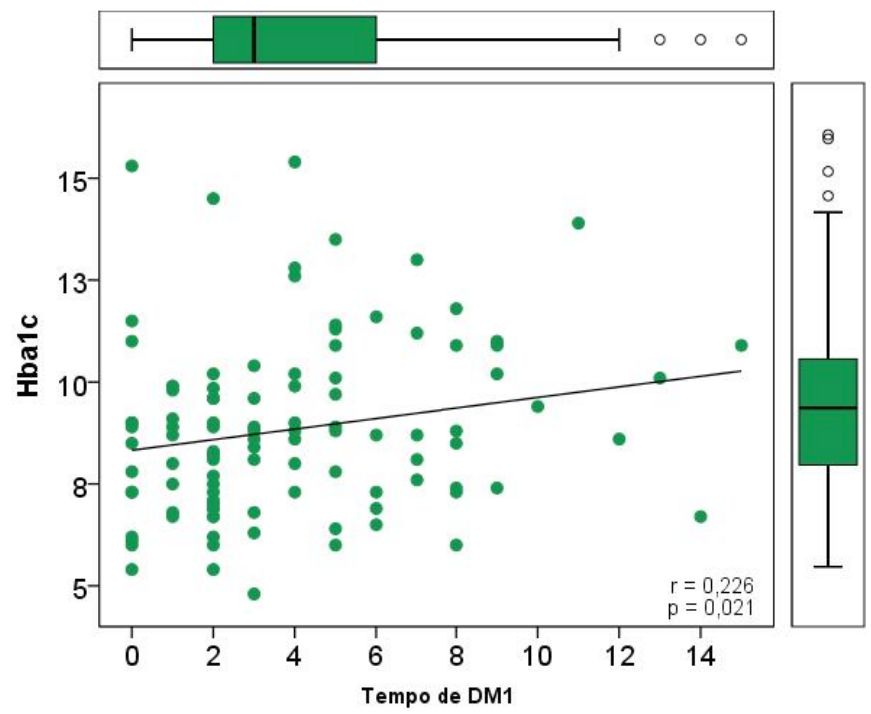

A dose total de insulina foi de $0,96 \mathrm{UI} / \mathrm{kg} / \mathrm{dia}$ (DP: $\pm 0,38$ ), sendo $0,55 \pm 0,27 \mathrm{UI} / \mathrm{kg} / \mathrm{dia}(57,2 \%)$ em forma basal e 0,42 $\pm 0,19 \mathrm{UI} / \mathrm{kg} / \mathrm{dia}(42,7 \%)$ em forma de bolus. Não houve correlação entre HbA1c e a relação basal/bolus $(p=0,187)$, porém, este relacionou-se à dose total de insulina $(p=0,00)$. A dose total de insulina não foi relacionada ao SDS IMC $(p=0,323)$ (Tabela 1).

Doses de insulina variaram a depender da faixa etária. Foi verificado maior dose de insulina total em adolescentes $(1,02$ $\mathrm{UI} / \mathrm{kg} / \mathrm{dia})$ em relação aos pré-escolares $(0,69 \mathrm{UI} / \mathrm{kg} / \mathrm{dia}$; $p=0,024)$. Maior dose bolus nos adolescentes $(0,45 \mathrm{UI} / \mathrm{kg} /$ dia) em relação aos pré-escolares $(0,27 \mathrm{UI} / \mathrm{kg} / \mathrm{dia} ; p=0,007)$. $\mathrm{Na}$ comparação entre grupos etários, não houve diferença entre doses basais $(p=0,233)$ (Tabela 1$)$.

Análogos de insulina de ação lenta foram utilizados em 65,5\% dos indivíduos, de ação ultrarrápida em 68,1\%, NPH em $34,5 \%$ e regular em $31,9 \%$ (Tabela 2 ).

Os meios de aplicação utilizados foram: caneta (65,3\%), seringa $(30,5 \%)$ ou ambos (4,2\%). A média de reutilização da agulha para aplicação foi de 7,6 vezes (DP: $\pm 3,37$ ), sendo mais frequente nos adolescentes e nos adultos $(9 \pm 16$ vezes $\mathrm{e}$ $14 \pm 20$ vezes, respectivamente; $p=0,01$ ) (Tabela 1). Pacientes reaproveitavam as agulhas de aplicação em $80 \%$ dos casos pelo custo acarretado ao utilizá-las em maior quantidade e, em $20 \%$ dos casos, pela conveniência de utilizar a mesma agulha de aplicação.

Tabela 1. Dados gerais.

\begin{tabular}{|c|c|c|c|}
\hline Variáveis & Média \pm DP & Mediana (Mín - Máx) & $\mathrm{p}^{*}$ \\
\hline \multicolumn{4}{|c|}{ Idade ao diagnóstico de DM1 e tempo de DM1 (anos) } \\
\hline Idade ao diagnóstico de DM1 & $7,9 \pm 4,4$ & $9(0-17)$ & \\
\hline Tempo de DM1 & $3,6 \pm 3,3$ & $3(0-15)$ & \\
\hline \multicolumn{4}{|l|}{ Dados antropométricos } \\
\hline SDS estatura & & & 0,252 \\
\hline Pré-escolar & $0,05 \pm-0,05$ & $0,68(-0,83-1,42)$ & \\
\hline Escolar & $-0,11 \pm 0,18$ & $1,43(-2,97-2,3)$ & \\
\hline Adolescente & $-0,54 \pm-0,59$ & $1,11(-4,6-1,61)$ & \\
\hline Adultos & $-0,28 \pm-0,28$ & $1,44(-1,3-0,74)$ & \\
\hline Total & $-0,38 \pm-0,3$ & $1,14(-4,6-2,3)$ & \\
\hline SDS IMC & & & $<0,001$ \\
\hline Pré-escolar & $1,75 \pm 1,46$ & $0,97(0,64-3,23)$ & \\
\hline Escolar & $0,54 \pm 0,42$ & $0,91(-0,8-2,17)$ & \\
\hline Adolescente & $-0,3 \pm 0,2$ & $4,8(-39-2,59)$ & \\
\hline Adultos & $0,99 \pm 0,99$ & $0,11(0,91-1,06)$ & \\
\hline Total & $0,15 \pm 0,5$ & $4,01(-39-3,23)$ & \\
\hline HbA1C por faixa etária (\%) & & & 0,186 \\
\hline Pré-escolar & $7,85 \pm 8$ & $0,99(6,1-9)$ & \\
\hline Escolar & $8,15 \pm 8,3$ & $1,19(6-10,2)$ & \\
\hline
\end{tabular}

Continua. 
Conclusão.

Tabela 1. Dados gerais.

\begin{tabular}{|c|c|c|c|}
\hline Variáveis & Média \pm DP & Mediana (Mín - Máx) & $\mathrm{p}^{*}$ \\
\hline HbA1C por faixa etária $(\%)$ & & & 0,186 \\
\hline Adolescente & $9,06 \pm 8,8$ & $2,39(4,8-15,4)$ & \\
\hline Adultos & $10,19 \pm 10,2$ & $2,3(6,7-13)$ & \\
\hline Total & $8,84 \pm 8,7$ & $2,16(4,8-15,4)$ & \\
\hline Doses de insulina por faixa etária (UI/Kg/dia) & & & 0,024 \\
\hline Pré-escolar & $0,69 \pm 0,75$ & $0,27(0,2-1,11)$ & \\
\hline Escolar & $0,9 \pm 0,93$ & $0,32(0,3-1,37)$ & \\
\hline Adolescente & $1,02 \pm 1$ & $0,39(0,29-2)$ & \\
\hline Adultos & $1,09 \pm 1,08$ & $0,45(0,46-1,7)$ & \\
\hline Total & $0,96 \pm 0,96$ & $0,38(0,2-2)$ & \\
\hline Dose basal de insulina & & & 0,233 \\
\hline Pré-escolar & $0,42 \pm 0,44$ & $0,17(0,15-0,76)$ & \\
\hline Escolar & $0,52 \pm 0,52$ & $0,22(0,1-0,84)$ & \\
\hline Adolescente & $0,59 \pm 0,52$ & $0,29(0,16-1,8)$ & \\
\hline Adultos & $0,59 \pm 0,61$ & $0,32(0,14-1,03)$ & \\
\hline Total & $0,55 \pm 0,51$ & $0,27(0,1-1,8)$ & \\
\hline Dose bolus de insulina & & & 0,007 \\
\hline Pré-escolar & $0,27 \pm 0,27$ & $0,13(0,05-0,54)$ & \\
\hline Escolar & $0,39 \pm 0,4$ & $0,16(0,13-0,72)$ & \\
\hline Adolescente & $0,45 \pm 0,41$ & $0,19(0,13-0,95)$ & \\
\hline Adultos & $0,49 \pm 0,45$ & $0,14(0,32-0,67)$ & \\
\hline Total & $0,42 \pm 0,4$ & $0,19(0,05-0,95)$ & \\
\hline Agulhas de aplicação & & & 0,01 \\
\hline \multicolumn{4}{|l|}{ Quantas vezes utiliza mesma agulha } \\
\hline \multicolumn{4}{|l|}{ Lactente } \\
\hline Pré-escolar & $3 \pm 3$ & $2(1-8)$ & \\
\hline Escolar & $5 \pm 3$ & $5(2-28)$ & \\
\hline Adolescente & $9 \pm 4$ & $16(1-90)$ & \\
\hline Adultos & $14 \pm 3$ & $20(3-49)$ & \\
\hline Total & $8 \pm 4$ & $13(1-90)$ & \\
\hline
\end{tabular}

* teste de Kruskal-Wallis.

Tabela 2. Tipos de insulinas.

\begin{tabular}{lll}
\hline Tipo de insulina lenta & $\mathrm{N}$ & $\%$ \\
\hline NPH & 41 & $34,5 \%$ \\
Glargina & 52 & $43,7 \%$ \\
Detemir & 20 & $16,8 \%$ \\
Degludeca & 6 & $5 \%$ \\
Total & 119 & $100,0 \%$ \\
Tipo de insulina rápida & & \\
Regular & 38 & $31,9 \%$ \\
Análogo Ultra-rápido & 81 & $68,1 \%$ \\
Total & 119 & $100 \%$ \\
\hline
\end{tabular}


A agulha mais utilizada foi a $4 \mathrm{~mm}(36,2 \%)$ e a menos utilizada foi a agulha de $8 \mathrm{~mm}$, com uso em 5\% dos escolares e em $14,5 \%$ dos adultos. Do total de participantes, $31 \%$ não sabiam o tamanho de sua agulha de aplicação. Não houve diferença significativa entre tamanho da agulha e HbA1c $(p=0,826)$. Os diferentes tipos de agulhas também não foram relacionados à lipo-hipertrofia $(p=0,833)$. As doses total, basal e bolus não diferiram de acordo com tamanho de agulhas de aplicação de insulina $(p=0,183 ; p=0,411$; $p=0,158$, respectivamente).

Os injetáveis eram descartados em Unidade Básica de Saúde em $63 \%$ dos casos e em $35,3 \%$ seu descarte ocorria em lixo comum.

Apenas 54,2\% realizavam assepsia prévia à aplicação de insulina, sendo o álcool o produto asséptico utilizado preferencialmente $(67 \%)$.
Total de $81 \%$ dos participantes relatou realizar prega subcutânea para aplicação de insulina, enquanto 15,5\% não realizavam.

Do total dos pacientes, a região glútea foi a menos utilizada para aplicação da medicação $(52,1 \%$ não utilizavam essa região), seguida da região abdominal em 27,4\% dos participantes. Somente $43,4 \%$ dos adolescentes utilizavam a região glútea para aplicação, sendo os pré-escolares os que mais a utilizavam $(73,3 \% ; p=0,017)$. Apenas $31,1 \%$ faziam rodízio em todos sítios de aplicação indicados e 39,5\% referiram utilizar pelo menos 3 regiões de aplicação.

A auto aplicação de insulina ocorreu em 42,7 \% dos casos, sendo 9,5\% escolares com supervisão dos responsáveis; $59,2 \%$ dos adolescentes e $60 \%$ dos adultos (Tabela 3 ). Houve predomínio da aplicação imediatamente antes das refeições (40,2\%), seguido de aplicação 10 minutos antes das refeições e 30 minutos antes das refeições em 18,8\% ambos.

Tabela 3. Aplicação insulina.

\begin{tabular}{llllllll}
\hline & & Pré-escolar & Escolar & Adolescente & Adulto & Total \\
\hline Quem aplica & Paciente & $\mathrm{N}$ & 0 & 2 & 45 & 3 & 50 \\
insulina & & $\%$ & $0,0 \%$ & $9,5 \%$ & $59,2 \%$ & $60,0 \%$ & $42,7 \%$ \\
& \multirow{2}{*}{ Responsável } & $\mathrm{N}$ & 15 & 12 & 3 & 0 & 30 \\
& & $\%$ & $100,0 \%$ & $57,1 \%$ & $3,9 \%$ & $0,0 \%$ & $25,6 \%$ \\
& \multirow{3}{*}{ Ambos } & $\mathrm{N}$ & 0 & 7 & 28 & 2 & 37 \\
& & $\%$ & $0,0 \%$ & $33,3 \%$ & $36,8 \%$ & $40,0 \%$ & $31,6 \%$ \\
\hline
\end{tabular}

Enquanto a lipo-hipertrofia foi diagnosticada pelo médico em $47,8 \%$ dos pacientes, apenas $34,7 \%$ destes referiam sua presença. Lipo-hipertrofia não mostrou correlação com dose de insulina total $(p=0,559)$, basal $(p=0,680)$ ou bolus ( $p=$ $0,569)$. Ela também não se relacionou à idade do paciente ( $p=$ $0,223)$ como também à idade do $\operatorname{DM} 1(p=0,083)$.

\section{DISCUSSÃO}

Foi avaliado nesse estudo o conhecimento acerca das práticas de injetáveis em pacientes portadores de DM1. Todos os pacientes utilizavam insulina injetável, sob forma de caneta $(65,3 \%)$, de seringas com agulha $(30,5 \%)$ ou ambos $(4,2 \%)$. Dados semelhantes são vistos em estudos brasileiros, porém, com menor número de usuários de caneta para aplicação. ${ }^{11}$ Estudos multicêntricos referem que não há diferença entre segurança e eficácia de acordo com aplicação de insulina sob forma de caneta ou de seringas. Os pacientes, porém, relatam maior preferência pelo uso da caneta para aplicação. ${ }^{12}$

A média de dose total, basal e bolus foi semelhante a outros estudos. ${ }^{13}$ Strich e colaboradores, porém, referem melhor controle glicêmico em pacientes com menor dose basal em relação à dose bolus de insulina. ${ }^{14}$

Valores de HbA1c não diferiram entre tamanho das agulhas, porém, foram encontrados valores maiores em pacientes adolescentes e adultos, com correlação ao tempo de doença. A média da HbA1c encontrada foi menor que a relatada em estudo multicêntrico nacional $(8,84$ vs 9,25$)$, no qual, Gomes et al evidenciaram relação de $\mathrm{HbA} 1 \mathrm{c}$ com sexo feminino e idade. $^{10}$

Apesar de uma média de 3,59 anos de diagnóstico, muitos pacientes não dominavam técnicas dos seus injetáveis, com ausência de rodízio de aplicação em todos os grupos indicados para injeção subcutânea $(78,9 \%)$ e com alto número de repetição de uso de agulhas $(7,6 \pm 3,37)$, encontrado com maior frequência entre adolescentes e adultos. A baixa frequência de rodízio de sítios de aplicação também é relatada por Strauss et al. com aplicação nos quatro sítios variando de $19,4 \%$ a $37,4 \%$ de acordo com tamanho da agulha utilizada para aplicação. ${ }^{7}$

Esse grande número de reutilização de agulhas se deve, em $80 \%$ das vezes, ao custo financeiro acarretado em relação ao uso único. Dados concordam com estudos semelhantes já realizados que demonstram associação de reutilização de agulhas a complicações como infecções e lipo-hipertrofia. ${ }^{15,16}$

O grupo muscular mais utilizado para aplicação foi o membro superior $(90,6 \%)$ em contraste com estudo multicêntrico internacional realizado após aplicação ITQ, que demonstrou maior número de aplicações em abdome..$^{6,7}$ 
No grupo de adolescentes houve maior reutilização de agulhas e menor rodízio de aplicação. Os pré-escolares obtiveram melhores resultados, provavelmente por maior comprometimento de seus responsáveis no cuidado da doença.

A agulha mais utilizada foi a de $4 \mathrm{~mm}$, tanto em crianças como em adultos. Essa agulha promove menos dor, controle glicêmico equivalente e menor perda de insulina quando comparada à agulha de $6 \mathrm{~mm}$ e de $8 \mathrm{~mm}$ em estudos anteriores. ${ }^{16}$ Publicações anteriores relatam menor prevalência de lipo-hipertrofia em pacientes com agulha de $4 \mathrm{~mm}$, o que não foi confirmado no presente estudo provavelmente devido ao tamanho da amostra. ${ }^{7,17,18} \mathrm{Em}$ alguns estudos, foram encontradas menores doses totais de insulina em pacientes com uso da agulha de $4 \mathrm{~mm}$, o que também não foi encontrado atualmente nessa análise.?

Total de $45,7 \%$ dos pacientes realizavam auto aplicação de insulina. Esse número foi maior em adolescentes e adultos, demonstrando que o cuidado da doença foi assumido pelo próprio paciente.

Outro achado importante foi descarte impróprio de injetáveis em lixo comum $(35,3 \%)$. Esse ato aumenta riscos de contaminações e perfurações cutâneas e deve ser amplamente desestimulado. Deve, portanto, existir um planejamento adequado para eliminação de resíduos em Unidades de Saúde próximas. ${ }^{19,20}$

Foi encontrada lipo-hipertrofia em 47,3 \% dos pacientes, porém alguns desconheciam sua presença. Ela não foi

\section{REFERÊNCIAS}

1. Skyler JS (Ed.). Medical Management of Type 1 Diabetes. 3. ed. Alexandria (VA): American Diabetes Association; 1998.

2. Diabetes Control and Complication Trial Research group - DCCT. The effect of intensive treatment of diabetes on the development and progression of long-term complications in insulin-dependent diabetes mellitus. N Engl J Med. 1993;329:977-86.

3. United Kingdom Prospective Diabetes Study Group (UKPDS Group). Intensive blood glucose control with sulphonylureas or insulin compared with conventional treatment and risk of complications in patients with type 2 diabetes (UKPDS 33). Lancet. 1998;352:837-53.

4. Brasil. Ministério da Saúde. Secretaria de Políticas de Saúde. Departamento de Atenção Básica. Saúde da criança: acompanhamento do crescimento e desenvolvimento infantil. Brasília: Ministério da Saúde; 2002.

5. Conwell LS, Pope E, Artiles AM, Mohanta A, Daneman A, Daneman D. Dermatological complications of continuous subcutaneous insulin infusion in children and adolescents. J Pediatr. 2008;152:622-8.

6. Frid AH, Kreugel G, Grassi G, Halimi S, Hicks D, Hirsch LJ, et al. New insulin delivery recommendations. Mayo Clin Proc. 2016;91(9):1231-55.

7. Frid AH, Hirsch LJ, Menchior AR, Morel DR, Strauss KW. Worldwide injection technique questionnaire study: injecting associada à idade, dose total, dose basal, dose bolus de insulina, tamanho da agulha nem ao tempo de diagnóstico. Esses dados discordam com outros estudos que demonstram associação de lipo-hipertrofia com tempo de doença, ausência de rodízio de aplicação, maior número de reutilização de agulhas, maior dose de insulina e maior HbA1c. ${ }^{16,18}$

Como limitações do estudo, podem-se citar a pequena amostra de pacientes, como também não foram incluídas para análise algumas variáveis como renda, escolaridade e uso de contagem de carboidratos.

\section{CONCLUSÃO}

Esse estudo permitiu conhecimento sobre uso de insulina e seus insumos em pacientes com DM1. Através dos resultados analisados nota-se que ainda há falha sobre uso de injetáveis em pacientes diabéticos. O presente estudo mostra que mesmo os que são orientados nesse aspecto, ainda não realizam todos os tópicos relacionados adequadamente.

O processo educativo deve acontecer de forma contínua na qual os profissionais, pacientes, familiares e acompanhantes aprendam sobre diabetes e possam conviver melhor com a doença, evitando, assim, desfechos desfavoráveis.

Dados avaliados reforçam, portanto, a necessidade de acompanhamento intensivo dos profissionais de saúde para melhor transmissão do conhecimento ao portador de DM1 para obter melhores desfechos relacionados a sua saúde.

complications and the role of the professional. Mayo Clin Proc. 2016;91(9):1224-30.

8. Sociedade Brasileira de Diabetes. Posicionamento oficial SBD ${ }^{\circ}$ 01/2017: recomendações sobre o tratamento injetável do diabetes: insulinas e incretinas. Turatti, L.A. São Paulo: Sociedade Brasileira de Diabetes; 2017.

9. Oliveira JE, Vencio S (organizadores). Diretrizes da Sociedade Brasileira de Diabetes (2015-2016). São Paulo: AC Farmacêutica; 2016.

10. Gomes MB, Cobas RA, Matheus AS, Tannus LR, Negrato CA, Rodacki M, et al. Regional differences in clinical care among patients with type 1 diabetes in Brazil: Brazilian Type 1 Diabetes Study Group. Diabetol Metab Syndr. 2012;4:44.

11. Souza CR, Zanetti ML. Administração de insulina: uma abordagem fundamental na educação em diabetes. Rev Esc Enf USP. 2000;34(3):264-70.

12. Korytkowski M, Bell D, Jacobsen C, Suwannasari R; FlexPen Study Team. A multicenter, randomized, open-label, comparative, two-period crossover trial of preference, efficacy, and safety profiles of a prefilled, disposable pen and conventional vial/syringe for insulin injection in patients with type 1 or 2 diabetes mellitus. Clin Ther. 2003;25(11):2836-48.

13. Bangstad HJ, Danne T, Deeb L, Jarosz-Chobot P, Urakami T, 
Hanas R. Insulin treatment in children and adolescents with diabetes. Pediatr Diabetes. 2009;10(Supl 12):82-99.

14. Strich D, Balagour L, Shenker J, Gilis D. Lower basal insulin dose is associated with better control in type 1 diabetes. J Pediatr. 2017;182:133-6.

15. Kreugel G, Keers JC, Kerstens MN, Wolffenbuttel BH. Randomized trial on the influence of the length of two insulin pen needles on glycemic control and patient preference in obese patients with diabetes. Diabetes Technol Ther. 2011;13(7):733-41.

16. Kalra S, Mithal A, Sahay R, John M, Unnikrishnan AG, Saboo $\mathrm{B}$, et al. Indian Injection Technique Study: injecting complications, education, and the health care professional. Diabetes Ther. 2017;8(3):659-72.
17. WangW, Guo X, Shen G, Wei Z, Liu J, Hirsch L, et al. Skin and subcutaneous thickness at insulin injection sites in Chinese patients with diabetes: clinical implications. Diabetes Metab. 2016;42(5):374-7.

18. Ji L, Sun Z, Li Q, Qin G, Wei Z, Liu J, et al. Lipohypertrophy in China: prevalence, risk factors, insulin consumption and clinical impact. Diabetes Technol Ther. 2017;19(1):61-7.

19. Silva EN, Santana PS, Palmeira CL. Descarte de seringas e agulhas por pacientes com Diabetes Mellitus. Revista Enfermagem Contemporânea. 2013;2(1):82-102.

20. Costa JA, Balga RS, Alfenas RC, Cotta RM. Promoção da saúde e diabetes: discutindo a adesão e a motivação de indivíduos diabéticos participantes de programas de saúde. Cien Saude Colet. 2011;16(3):2001-9.

\section{Como citar:}

Fernandes PM, Lima LP, Carvalho AB, Aragão LF, Sousa MS, Montenegro RM Junior, et al. Avaliação do uso de injetáveis em portadores de Diabetes Mellitus tipo 1 acompanhados em ambulatório de referência. Rev Med UFC. 2018 out-dez;58(4):26-32. 